\title{
SOBOLEV VERSUS HÖLDER MINIMIZERS FOR THE DEGENERATE FRACTIONAL p-LAPLACIAN
}

\author{
ANTONIO IANNIZZOTTO, SUNRA MOSCONI, AND MARCO SQUASSINA
}

\begin{abstract}
We consider a nonlinear pseudo-differential equation driven by the fractional $p$ Laplacian $(-\Delta)_{p}^{s}$ with $s \in(0,1)$ and $p \geqslant 2$ (degenerate case), under Dirichlet type conditions in a smooth domain $\Omega$. We prove that local minimizers of the associated energy functional in the fractional Sobolev space $W_{0}^{s, p}(\Omega)$ and in the weighted Hölder space $C_{s}^{0}(\bar{\Omega})$, respectively, do coincide.
\end{abstract}

\section{IntRoduction AND MAIN RESUlt}

The 'Sobolev versus Hölder minimizers problem' is a classical one in nonlinear analysis, arising from the application of variational methods to boundary value problems of the following general type:

$$
\left\{\begin{array}{l}
L u=f(x, u) \quad \text { in } \Omega \\
u \in \mathcal{W} .
\end{array}\right.
$$

Here $\Omega \subset \mathbb{R}^{N}$ is a (generally bounded and smooth) domain, $\mathcal{W}$ is a Sobolev-type function space defined on $\Omega$, incorporating some boundary condition, $L: \mathcal{W} \rightarrow \mathcal{W}^{*}$ is a (linear or nonlinear) elliptic differential operator in divergence form, and the reaction $f: \Omega \times \mathbb{R} \rightarrow \mathbb{R}$ is a Carathéodory mapping satisfying suitable growth conditions. In many relevant cases, weak solutions of problem (1.1) coincide with critical points of an energy functional $J \in C^{1}(\mathcal{W})$ of the form

$$
J(u)=\frac{\|u\|_{\mathcal{W}}^{p}}{p}-\int_{\Omega} F(x, u) d x
$$

where $p>1$ and $F(x, \cdot)$ denotes a primitive of $f(x, \cdot)$. Among critical points, local minimizers play a special rôle, as they are the starting point to apply mountain pass or minimax schemes, as well as Morse-theoretic arguments aimed at multiplicity results.

In order to localize solutions of (1.1), truncations of the reaction are often employed: as a typical example, when $f(\cdot, 0)=0$, positive solutions are detected by using a modified functional $J_{+}$ defined as in (1.2), but with $f$ replaced by $f_{+}(x, t)=f\left(x, t^{+}\right)$(here $t^{+}$denotes the positive part of $t \in \mathbb{R})$. Alternatively, one may truncate $f(x, \cdot)$ outside a sub-solution and a super-solution of (1.1) in order to 'trap' solutions within a given functional interval. Among many examples, we refer the reader to the classical works $[1,23,27]$. In all these cases natural constraints are employed, so that weak comparison arguments ensure that critical points of the truncated functionals are critical points of $J$ as well. A drawback of the truncation method is that the topological nature of such critical points is a priori lost in the process, along with the valuable information that can be derived from it: in particular, it is not a-priori ensured that local minimizers of the truncated functionals are minimizers of $J$. For instance, this is the case for

2010 Mathematics Subject Classification. 35D10, 35R11, 47G20.

Key words and phrases. Fractional p-Laplacian, Fractional Sobolev spaces, Weighted Hölder regularity, Boundary regularity. 
the truncation at 0 , as $J$ and $J_{+}$only agree on the positive cone $\mathcal{W}_{+}$, which in general has an empty interior.

In [4], Brezis and Nirenberg proposed an answer to this issue for the Dirichlet problem with $L=-\Delta$ and $\mathcal{W}=H_{0}^{1}(\Omega)$, by proving that local minimizers of $J$ in $H_{0}^{1}(\Omega)$ coincide with those in the space $\mathcal{C}=C^{1}(\bar{\Omega})$ (whose positive cone has a nonempty interior). Such result relies on classical elliptic regularity theory, as well as on the linearity of the operator (coincidence is first proved for 0 , and then extended to any minimizer $u$ by translation). A key point is that minimizers of $J$ on closed balls in $H_{0}^{1}(\Omega)$ solve a problem of the form (1.1), involving a Lagrange's multiplier as well.

When nonlinear operators are considered, the question becomes more involved. In [13], Garcìa Azorero, Peral Alonso and Manfredi extended the coincidence result to the $p$-Laplacian operator $L=-\Delta_{p}$, with $\mathcal{W}=W_{0}^{1, p}(\Omega)(p>1)$ and again $\mathcal{C}=C^{1}(\bar{\Omega})$. In this case, to deal with nonzero minimizers, a simple translation would not work, but the authors overcame such difficulty by employing the nonlinear regularity theory of Lieberman [22] for a more general operator than $-\Delta_{p}$. Since then, 'Sobolev versus Hölder' results were proved for a number of (1.1)-type problems involving several operators (both linear and nonlinear) and boundary conditions (see for instance $[9,14,20]$ ). In particular, we mention the approach of Brock, Iturriaga and Ubilla [5], where constrained minimization of $J$ is performed on balls in $L^{q}(\Omega)\left(q<p^{*}\right)$, so that the modified equation is still of $p$-Laplacian type, though involving an additional power term (see also [10]).

When it comes to nonlocal operators of fractional order, problem (1.1) is naturally set in a fractional Sobolev space $\mathcal{W}=H_{0}^{s}(\Omega)$, but $C^{1}$-regularity up to the boundary is not to be expected any more. For instance, given $s \in(0,1)$, the function $\left(1-|x|^{2}\right)_{+}^{s}$ solves

$$
\begin{cases}(-\Delta)^{s} u=1 & \text { in } B_{1} \\ u=0 & \text { in } \mathbb{R}^{N} \backslash B_{1},\end{cases}
$$

but clearly $|\nabla u|$ blows up near the boundary. The issue, moreover, does not only involve the boundary behaviour: for $f \in L^{\infty}\left(\mathbb{R}^{N}\right)$, the optimal interior regularity for solutions of $(-\Delta)^{s} u=f$ is $C^{2 s}$ when $s \neq 1 / 2$, so that when $s<1 / 2$ we cannot expect even Lipschitz continuity in the interior (see [25]). In the fractional framework, the natural function space to work with is constructed through a weighted Hölder regularity condition, namely assuming that $u / \mathrm{d}_{\Omega}^{s}$ admits a continuous extension to $\bar{\Omega}$, where $\mathrm{d}_{\Omega}^{s}(x)=\operatorname{dist}\left(x, \mathbb{R}^{N} \backslash \Omega\right)^{s}$. We denote by $\mathcal{C}=C_{s}^{0}(\bar{\Omega})$ the space of such $u$ 's, equipped with the norm $\|u\|_{C_{s}^{0}}=\sup _{\Omega}|u| / \mathrm{d}_{\Omega}^{s}$, while we set $L=(-\Delta)^{s}$ (the fractional Laplacian of order $\left.s \in(0,1)\right)$ and $\mathcal{W}=H_{0}^{s}(\Omega)$. In [16], equivalence of minimizers for $J$ in $\mathcal{W}$ and in $\mathcal{C}$ was proved following the approach of [4] (see also [2,11] for similar results).

In this work we propose a 'Sobolev versus Hölder' result for a nonlinear nonlocal equation driven by the degenerate fractional $p$-Laplacian, namely the nonlinear extension of $(-\Delta)^{s}$. For any $s \in(0,1), p \geqslant 1, N>p s$ we define the Gagliardo (semi-)norm of a measurable function $u: \mathbb{R}^{N} \rightarrow \mathbb{R}$ as

$$
\|u\|_{s, p}^{p}=\iint_{\mathbb{R}^{2 N}}|u(x)-u(y)|^{p} d \mu
$$

where we use the abbreviated notation

$$
d \mu=\frac{d x d y}{|x-y|^{N+p s}}
$$

Further, let $\Omega \subset \mathbb{R}^{N}$ be a bounded domain with a $C^{1,1}$-boundary and set

$$
W_{0}^{s, p}(\Omega)=\left\{u \in L^{p}\left(\mathbb{R}^{N}\right):\|u\|_{s, p}<\infty, u=0 \text { a.e. in } \mathbb{R}^{N} \backslash \Omega\right\} .
$$


The space $W_{0}^{s, p}(\Omega)$, endowed with the norm $\|\cdot\|_{s, p}$, is a separable, uniformly convex Banach space with dual denoted by $W^{-s, p^{\prime}}(\Omega)$. The embedding $W_{0}^{s, p}(\Omega) \hookrightarrow L^{q}(\Omega)$ is continuous for all $q \in\left[1, p_{s}^{*}\right]$ and compact for all $q \in\left[1, p_{s}^{*}\right)$, where

$$
p_{s}^{*}=\frac{N p}{N-p s}
$$

denotes the fractional Sobolev exponent. We define the fractional $p$-Laplacian as an operator $(-\Delta)_{p}^{s}: W_{0}^{s, p}(\Omega) \rightarrow W^{-s, p^{\prime}}(\Omega)$ given by

$$
\left\langle(-\Delta)_{p}^{s} u, \varphi\right\rangle=\iint_{\mathbb{R}^{2 N}}(u(x)-u(y))^{p-1}(\varphi(x)-\varphi(y)) d \mu,
$$

i.e., $(-\Delta)_{p}^{s}$ is the Fréchet derivative of $u \mapsto\|u\|_{s, p}^{p} / p$ (see [15] for details). Note that $(-\Delta)_{p}^{s}$ is both nonlinear and nonlocal. We will consider the following Dirichlet problem:

$$
\begin{cases}(-\Delta)_{p}^{s} u=f(x, u) & \text { in } \Omega \\ u=0 & \text { in } \mathbb{R}^{N} \backslash \Omega,\end{cases}
$$

$f: \Omega \times \mathbb{R} \rightarrow \mathbb{R}$ is a Carathéodory mapping obeying the following at most critical growth condition for a.e. $x \in \Omega$ and all $t \in \mathbb{R}$ :

$$
|f(x, t)| \leqslant C_{0}\left(1+|t|^{p_{s}^{*}-1}\right) \quad\left(C_{0}>0\right) .
$$

According to the general formula (1.2), the energy functional for problem (1.3) is $J \in C^{1}\left(W_{0}^{s, p}(\Omega)\right)$, defined by

with

$$
J(u)=\frac{\|u\|_{s, p}^{p}}{p}-\int_{\Omega} F(x, u) d x
$$

$$
F(x, t)=\int_{0}^{t} f(x, \tau) d \tau .
$$

In the present case, the rôle of the space $\mathcal{C}$ is played by the weighted Hölder space

$$
C_{s}^{0}(\bar{\Omega})=\left\{u \in C^{0}(\bar{\Omega}): \frac{u}{\mathrm{~d}_{\Omega}^{s}} \in C^{0}(\bar{\Omega})\right\}
$$

endowed with the norm

$$
\|u\|_{C_{s}^{0}}=\left\|\frac{u}{\mathrm{~d}_{\Omega}^{s}}\right\|_{\infty}
$$

Our main result is the following, proving coincidence of Sobolev and Hölder minimizers of $J$ :

Theorem 1.1. Let $p \geqslant 2, s \in(0,1), N>p s, \Omega \subseteq \mathbb{R}^{N}$ be a bounded domain with a $C^{1,1}$ boundary, $f: \Omega \times \mathbb{R} \rightarrow \mathbb{R}$ be a Carathéodory mapping satisfying (1.4). Then, for any $u_{0} \in$ $W_{0}^{s, p}(\Omega)$, the following are equivalent:

(i) there exists $\rho>0$ such that $J\left(u_{0}+v\right) \geqslant J\left(u_{0}\right)$ for all $v \in W_{0}^{s, p}(\Omega),\|v\|_{s, p} \leqslant \rho$;

(ii) there exists $\sigma>0$ such that $J\left(u_{0}+v\right) \geqslant J\left(u_{0}\right)$ for all $v \in W_{0}^{s, p}(\Omega) \cap C_{s}^{0}(\bar{\Omega}),\|v\|_{C_{s}^{0}} \leqslant \sigma$.

We make some comments on Theorem 1.1:

(a) Choice of the space. There are many reasons why $C_{s}^{0}(\bar{\Omega})$ is a natural choice where to settle this kind of result. Mainly, such choice is dictated by the results of [18] (see also $[17,18])$, where an a priori bound for solutions of $(1.3)$ in the space $C_{s}^{\alpha}(\bar{\Omega})=$ $\left\{u \in C^{\alpha}(\bar{\Omega}): u / \mathrm{d}_{\Omega}^{s} \in C^{\alpha}(\bar{\Omega})\right\}$ for some $\alpha>0$, which compactly embeds into $C_{s}^{0}(\bar{\Omega})$ (see Section 2 for details). Plus, in [7] (see also [21]) the following version of Hopf's lemma was proved: any solution $u \geqslant 0$ of (1.3) with non-negative right hand side either vanishes identically, or $u / \mathrm{d}_{\Omega}^{s} \geqslant c$ for some $c>0$. In particular, these signed solutions belongs to the interior of the non-negative cone in $C_{s}^{0}(\bar{\Omega})$. 
(b) Method of proof. Our strategy is more in the spirit of [5] rather than of [13], with constrained minimization on $L^{p_{s}^{*}}(\Omega)$-balls in order to deal with possibly critical problems, and employs as well a special monotonicity property of $(-\Delta)_{p}^{s}$ (see Section 3 for the detailed proof). Notice that in references such as $[4,5,13]$ only the implication (ii) $\Rightarrow(i)$ is considered, as the other one is trivial due to $C^{1}(\bar{\Omega}) \hookrightarrow W_{0}^{1, p}(\Omega)$. A typical feature of the nonlocal framework is that $C_{s}^{0}(\bar{\Omega})$ is not included in $W_{0}^{s, p}(\Omega)$, so we have to prove both implications.

(c) Applications. The semi-linear case $p=2$ of Theorem 1.1 proved in [16] has already been applied in a number of settings, see e.g. $[8,12,26]$ or the survey [24]. We believe that Theorem 1.1 will prove to be equally useful in the quasi-linear setting. Already in [15, Theorem 5.3], a multiplicity result for problem (1.3) was proved under the conjecture that a version of Theorem 1.1 holds: such result is now fully achieved. In Section 4, we will briefly describe an illustrative application.

(d) The singular case. We remark that our result is only proved in the degenerate (or superquadratic) case $p \geqslant 2$. This is due to the fact that the $C_{s}^{\alpha}(\bar{\Omega})$-regularity mentioned above has so far only been proved in this setting. For the singular case $p \in(1,2)$, the boundary regularity issue is therefore still open, nevertheless the corresponding case of Theorem 1.1 could be easily proven with only slight modifications, using Lemma 2.4 toghether with the putative singular counterpart of [19].

Notation. Throughout the paper we will use the short notation $a^{q}=|a|^{q-1} a$ for all $a \in \mathbb{R}$, $q \geqslant 1$. We will denote $\|\cdot\|_{q}$ the usual norm of $L^{q}(\Omega)$ for all $q \in[1, \infty]$. Finally, $C$ will denote several positive constants, only depending on the data of the problem.

\section{Preliminaries}

In this section we introduce some technical results which will be used in the proof of our main theorem. First, we recall that $u \in W_{0}^{s, p}(\Omega)$ is a (weak) solution of problem (1.3) iff for all $\varphi \in W_{0}^{s, p}(\Omega)$

$$
\left\langle(-\Delta)_{p}^{s} u, \varphi\right\rangle=\int_{\Omega} f(x, u) \varphi d x
$$

i.e., iff $J^{\prime}(u)=0$ in $W^{-s, p^{\prime}}(\Omega)$. We recall from [6, Theorem 3.3, Remark 3.8] the following $a$ priori bound for weak solutions of (1.3):

Lemma 2.1. There exists $\varepsilon_{0}=\varepsilon_{0}\left(N, p, s, C_{0}\right)>0$ such that if $u \in W_{0}^{s, p}(\Omega)$ solves (1.3) under the growth condition (1.4) and $K>0$ fulfills

$$
\int_{\{|u| \geqslant K\}}|u|^{p_{s}^{*}} d x<\varepsilon_{0}
$$

then $\|u\|_{\infty} \leqslant C$ with $C=C\left(N, p, s, C_{0},\|u\|_{s, p}, K\right)>0$.

The bound in Lemma 2.1 is not uniform in $\|u\|_{s, p}$, due to the critical growth in (1.4), therefore in order to prove equi-boundedness of a sequence $\left(u_{n}\right)_{n}$ of solutions to (1.3) one not only needs an a priori bound on $\left\|u_{n}\right\|_{s, p}$, but also an equi-integrability estimate. For strictly subcritical reactions the dependance on $K$ can be dropped, and the former is sufficient.

In addition to $C_{s}^{0}(\bar{\Omega})$ defined in the Introduction, we will also use the following weighted Hölder space (see [16] for details):

$$
C_{s}^{\alpha}(\bar{\Omega})=\left\{u \in C^{0}(\bar{\Omega}): \frac{u}{\mathrm{~d}_{\Omega}^{s}} \in C^{\alpha}(\bar{\Omega})\right\}, \quad(\alpha \in(0,1)),
$$


with norm

$$
\|u\|_{C_{s}^{\alpha}}=\|u\|_{C_{s}^{0}}+\sup _{x \neq y} \frac{\left|u(x) / \mathrm{d}_{\Omega}^{s}(x)-u(y) / \mathrm{d}_{\Omega}^{s}(y)\right|}{|x-y|^{\alpha}} .
$$

The embedding $C_{s}^{\alpha}(\bar{\Omega}) \hookrightarrow C_{s}^{0}(\bar{\Omega})$ is compact for all $\alpha \in(0,1)$. The space $C_{s}^{\alpha}(\bar{\Omega})$ is related to the global regularity theory for solutions of the following problem:

$$
\begin{cases}(-\Delta)_{p}^{s} u=g(x) & \text { in } \Omega \\ u=0 & \text { in } \mathbb{R}^{N} \backslash \Omega,\end{cases}
$$

with $g \in L^{\infty}(\Omega)$. Weak solutions are defined just as those of (1.3). From [19, Theorem 1.1] we have the following result:

Lemma 2.2. Let $p \geqslant 2$. Then, there exist $\alpha, C>0$, both depending on $\Omega, p$, and $s$, such that any weak solution $u \in W_{0}^{s, p}(\Omega)$ of (2.1) with $g \in L^{\infty}(\Omega)$ fulfills

$$
\|u\|_{C_{s}^{\alpha}} \leqslant C\|g\|_{\infty}^{\frac{1}{p-1}} \text {. }
$$

Another useful tool for our argument is the following monotonicity property of the fractional $p$-Laplacian, which we present separately in the degenerate and singular case.

Lemma 2.3. (degenerate case) Let $p \geqslant 2$. There exists $C=C(p)>0$ such that for all $u, v \in W_{0}^{s, p}(\Omega) \cap L^{\infty}(\Omega)$ and all $q \geqslant 1$

$$
\left\|(u-v)^{\frac{p+q-1}{p}}\right\|_{s, p}^{p} \leqslant C q^{p-1}\left\langle(-\Delta)_{p}^{s} u-(-\Delta)_{p}^{s} v,(u-v)^{q}\right\rangle .
$$

Proof. First we prove the following elementary inequality: for all $a, b, c, d \in \mathbb{R}$ such that $a-b=$ $c-d$ it holds

$$
\left|a^{\frac{p+q-1}{p}}-b^{\frac{p+q-1}{p}}\right|^{p} \leqslant C q^{p-1}\left(c^{p-1}-d^{p-1}\right)\left(a^{q}-b^{q}\right),
$$

with a constant $C=C(p)>0$ independent of $q$. We may assume that $a \geqslant b$ and $c \geqslant d$ since the former is equivalent to the latter, so that being $t \mapsto t^{r-1}$ increasing for all $r \geqslant 1$ all the factors of (2.2) are nonnegative. We apply [3, Lemma A.2] with $g(t)=t^{q}$ and

$$
G(t)=\int_{0}^{t}\left(g^{\prime}(\tau)\right)^{\frac{1}{p}} d \tau=\frac{p q^{\frac{1}{p}}}{p+q-1} t^{\frac{p+q-1}{p}}
$$

to get

$$
\left(a^{\frac{p+q-1}{p}}-b^{\frac{p+q-1}{p}}\right)^{p} \leqslant \frac{(p+q-1)^{p}}{p^{p} q}(a-b)^{p-1}\left(a^{q}-b^{q}\right) .
$$

Besides, $p \geqslant 2$ implies that $t \mapsto t^{p-2}$ is increasing on $\mathbb{R}_{+}$, hence

$$
\begin{aligned}
c^{p-1}-d^{p-1} & =(p-1) \int_{d}^{c}|t|^{p-2} d t \\
& \geqslant(p-1) \int_{-(c-d) / 2}^{(c-d) / 2}|t|^{p-2} d t \\
& =\frac{1}{2^{p-2}}(c-d)^{p-1} .
\end{aligned}
$$

Recalling that $a-b=c-d$ and concatenating with the previous inequality, we get

$$
\left(a^{\frac{p+q-1}{p}}-b^{\frac{p+q-1}{p}}\right)^{p} \leqslant \frac{2^{p-2}(p+q-1)^{p}}{p^{p} q}\left(c^{p-1}-d^{p-1}\right)\left(a^{q}-b^{q}\right),
$$


which yields (2.2). Now pick $u, v \in W_{0}^{s, p}(\Omega) \cap L^{\infty}(\Omega)$, so $(u-v)^{q} \in W_{0}^{s, p}(\Omega)$. By (2.2) with $a=u(x)-v(x), b=u(y)-v(y), c=u(x)-u(y)$ and $d=v(x)-v(y)$, we have

$$
\begin{aligned}
\left\|(u-v)^{\frac{p+q-1}{p}}\right\|_{s, p}^{p} & =\iint_{\mathbb{R}^{2 N}}\left|(u(x)-v(x))^{\frac{p+q-1}{p}}-(u(y)-v(y))^{\frac{p+q-1}{p}}\right|^{p} d \mu \\
& \leqslant C q^{p-1}\left\langle(-\Delta)_{p}^{s} u-(-\Delta)_{p}^{s} v,(u-v)^{q}\right\rangle,
\end{aligned}
$$

which proves the assertion.

In the singular case, the monotonicity is slightly different:

Lemma 2.4. (singular case) Let $p \in(1,2)$. Then, there exists $C=C(p)>0$ such that for all $u, v \in W_{0}^{s, p}(\Omega) \cap L^{\infty}(\Omega)$ and all $q \geqslant 1$

$$
\frac{\left\|(u-v)^{\frac{q+1}{2}}\right\|_{s, p}^{2}}{\left(\|u\|_{s, p}^{p}+\|v\|_{s, p}^{p}\right)^{2-p}} \leqslant C q\left\langle(-\Delta)_{p}^{s} u-(-\Delta)_{p}^{s} v,(u-v)^{q}\right\rangle .
$$

Proof. Again we start with an elementary inequality:

$$
\left|a^{\frac{q+1}{2}}-b^{\frac{q+1}{2}}\right|^{2} \leqslant C q\left(c^{p-1}-d^{p-1}\right)\left(a^{q}-b^{q}\right)\left(c^{2}+d^{2}\right)^{\frac{2-p}{2}}
$$

for all $a, b, c, d \in \mathbb{R}$ such that $a-b=c-d$, with a constant $C=C(p)>0$ independent of $q$. As in the previous proof, we may assume that $a \geqslant b$ and $c \geqslant d$. By the Cauchy-Schwartz inequality and the assumption $a-b=c-d$, we have

$$
\begin{aligned}
{\left[a^{\frac{q+1}{2}}-b^{\frac{q+1}{2}}\right]^{2} } & =\left[\frac{q+1}{2} \int_{b}^{a}|t|^{\frac{q-1}{2}} d t\right]^{2} \\
& \leqslant \frac{(q+1)^{2}}{4} \int_{b}^{a}|t|^{q-1} d t(a-b) \\
& =\frac{(q+1)^{2}}{4 q}\left(a^{q}-b^{q}\right)(c-d) .
\end{aligned}
$$

On the other hand, $|t| \leqslant\left(c^{2}+d^{2}\right)^{\frac{1}{2}}$ for all $t \in[d, c]$, which, along with $p<2$, implies for all $t \in[d, c]$

$$
|t|^{2-p} \leqslant\left(c^{2}+d^{2}\right)^{\frac{2-p}{2}} .
$$

In turn, the latter implies

$$
\begin{aligned}
c-d & =\int_{d}^{c}|t|^{2-p}|t|^{p-2} d t \\
& \leqslant\left(c^{2}+d^{2}\right)^{\frac{2-p}{2}} \int_{d}^{c}|t|^{p-2} d t \\
& =\frac{1}{p-1}\left(c^{2}+d^{2}\right)^{\frac{2-p}{2}}\left(c^{p-1}-d^{p-1}\right),
\end{aligned}
$$

which inserted into (2.4) gives (2.3). Now pick $u, v \in W_{0}^{s, p}(\Omega) \cap L^{\infty}(\Omega)$ and set, for any $x, y \in \mathbb{R}^{N}, a=u(x)-v(x), b=u(y)-v(y), c=u(x)-u(y)$ and $d=v(x)-v(y)$. Using (2.3) 
and Hölder's inequality with exponents $2 / p$ and $2 /(2-p)$, we get

$$
\begin{aligned}
& \left\|(u-v)^{\frac{q+1}{2}}\right\|_{s, p}^{p}=\iint_{\mathbb{R}^{2 N}}\left|(u(x)-v(x))^{\frac{q+1}{2}}-(u(y)-v(y))^{\frac{q+1}{2}}\right|^{p} d \mu \\
& \leqslant C q^{\frac{p}{2}} \iint_{\mathbb{R}^{2 N}}\left[\frac{\left((u(x)-u(y))^{p-1}-(v(x)-v(y))^{p-1}\right)\left((u(x)-v(x))^{q}-(u(y)-v(y))^{q}\right)}{\left((u(x)-u(y))^{2}+(v(x)-v(y))^{2}\right)^{\frac{p-2}{2}}}\right]^{\frac{p}{2}} d \mu \\
& \leqslant C q^{\frac{p}{2}}\left(\left\langle(-\Delta)_{p}^{s} u-(-\Delta)_{p}^{s} v,(u-v)^{q}\right\rangle\right)^{\frac{p}{2}}\left(\|u\|_{s, p}^{p}+\|v\|_{s, p}^{p}\right)^{\frac{2-p}{2}}
\end{aligned}
$$

with a different $C=C(p)>0$, still independent of $q$. Raising to the power $2 / p$ we conclude.

\section{Proof of the MAIN RESUlt}

In this section we prove our main result:

Proof of Theorem 1.1. First we prove that $(i)$ implies $(i i)$. Assuming $(i)$, we have in particular $J^{\prime}\left(u_{0}\right)=0$ in $W^{-s, p^{\prime}}(\Omega)$, hence by Lemma $2.1 u_{0} \in L^{\infty}(\Omega)$. In turn, by (1.4) we have $f(\cdot, u) \in L^{\infty}(\Omega)$. Then, Lemma 2.2 implies $u \in C_{s}^{0}(\bar{\Omega})$.

We argue by contradiction, assuming that there exists a sequence $\left(u_{n}\right)_{n}$ in $W_{0}^{s, p}(\Omega) \cap C_{s}^{0}(\bar{\Omega})$ such that $u_{n} \rightarrow u_{0}$ in $C_{s}^{0}(\bar{\Omega})$ and $J\left(u_{n}\right)<J\left(u_{0}\right)$ for all $n \in \mathbb{N}$. Then we have $u_{n} \rightarrow u_{0}$ in $L^{\infty}(\Omega)$, hence

So we have

$$
\lim _{n} \int_{\Omega} F\left(x, u_{n}\right) d x=\int_{\Omega} F\left(x, u_{0}\right) d x
$$

$$
\begin{aligned}
\limsup _{n} \frac{\left\|u_{n}\right\|_{s, p}^{p}}{p} & =\limsup _{n}\left[J\left(u_{n}\right)+\int_{\Omega} F\left(x, u_{n}\right) d x\right] \\
& \leqslant J\left(u_{0}\right)+\int_{\Omega} F\left(x, u_{0}\right) d x=\frac{\left\|u_{0}\right\|_{s, p}^{p}}{p},
\end{aligned}
$$

in particular $\left(u_{n}\right)$ is bounded in $W_{0}^{s, p}(\Omega)$. Passing to a subsequence, we have $u_{n} \rightarrow u_{0}$ in $W_{0}^{s, p}(\Omega)$, hence

$$
\left\|u_{0}\right\|_{s, p} \leqslant \liminf _{n}\left\|u_{n}\right\|_{s, p} .
$$

By the uniform convexity of $W_{0}^{s, p}(\Omega)$ it is easily seen that the latter implies $u_{n} \rightarrow u_{0}$ (strongly) in $W_{0}^{s, p}(\Omega)$. Then, for all $n \in \mathbb{N}$ big enough we have $\left\|u_{n}-u_{0}\right\|_{s, p} \leqslant \rho$ along with $J\left(u_{n}\right)<J\left(u_{0}\right)$, a contradiction. Thus, (ii) holds.

Now we prove that $(i i)$ implies $(i)$. First note that, by $(i i)$, for all $\varphi \in W_{0}^{s, p}(\Omega) \cap C_{s}^{0}(\bar{\Omega})$ we have

$$
\left\langle J^{\prime}\left(u_{0}\right), \varphi\right\rangle \geqslant 0
$$

Since $W_{0}^{s, p}(\Omega) \cap C_{s}^{0}(\bar{\Omega})$ is a dense subspace of $W_{0}^{s, p}(\Omega)$, we have $J^{\prime}\left(u_{0}\right)=0$ in $W^{-s, p^{\prime}}(\Omega)$. As above, using Lemmas 2.1 and 2.2 we deduce that $u_{0} \in C_{s}^{0}(\bar{\Omega})$, in particular $u_{0} \in L^{\infty}(\Omega)$. Again we argue by contradiction, assuming that there exists a sequence $\left(\tilde{u}_{n}\right)_{n}$ in $W_{0}^{s, p}(\Omega)$ such that $\tilde{u}_{n} \rightarrow u_{0}$ in $W_{0}^{s, p}(\Omega)$ and $J\left(\tilde{u}_{n}\right)<J\left(u_{0}\right)$ for all $n \in \mathbb{N}$. Set for all $n \in \mathbb{N}$

$$
\delta_{n}:=\left\|\tilde{u}_{n}-u_{0}\right\|_{p_{s}^{*}}, \quad B_{n}=\left\{u \in W_{0}^{s, p}(\Omega):\left\|u-u_{0}\right\|_{p_{s}^{*}} \leqslant \delta_{n}\right\} .
$$

By the continuous embedding $W_{0}^{s, p}(\Omega) \hookrightarrow L^{p_{s}^{*}}(\Omega)$ we have $\delta_{n} \rightarrow 0$, and $B_{n}$ is a closed convex (hence, weakly closed) subset of $W_{0}^{s, p}(\Omega)$. Due to the critical growth in (1.4), we cannot directly minimize $J$ over $B_{n}$, so we introduce a suitable truncation. Set for all $t \in \mathbb{R}, \kappa>0$

$$
[t]_{\kappa}=\operatorname{sign}(t) \min \{|t|, \kappa\} .
$$


For all $u \in W_{0}^{s, p}(\Omega)$ we have by dominated convergence

$$
\lim _{\kappa \rightarrow+\infty} \int_{\Omega} \int_{0}^{u} f\left(x,[t]_{\kappa}\right) d t d x=\int_{\Omega} F(x, u) d x .
$$

Fix $n \in \mathbb{N}, \varepsilon_{n} \in\left(0, J\left(u_{0}\right)-J\left(\tilde{u}_{n}\right)\right)$. By (3.1) we can find $\kappa_{n}>\left\|u_{0}\right\|_{\infty}+1$ such that

$$
\left|\int_{\Omega} F_{n}\left(x, \tilde{u}_{n}\right) d x-\int_{\Omega} F\left(x, \tilde{u}_{n}\right) d x\right|<\varepsilon_{n},
$$

where we have set

$$
f_{n}(x, t):=f\left(x,[t]_{\kappa_{n}}\right), \quad F_{n}(x, t)=\int_{0}^{t} f_{n}(x, \tau) d \tau .
$$

Note that, by (1.4),

Set for all $u \in W_{0}^{s, p}(\Omega)$

$$
\left|f_{n}(x, t)\right| \leqslant C_{n}:=C_{0}\left(1+\kappa_{n}^{p_{s}^{*}-1}\right) .
$$

$$
J_{n}(u)=\frac{\|u\|_{s, p}^{p}}{p}-\int_{\Omega} F_{n}(x, u) d x,
$$

hence $J_{n} \in C^{1}\left(W_{0}^{s, p}(\Omega)\right)$, is sequentially weakly lower semi-continuous and, being $p>1$ and $\left|F_{n}(x, t)\right| \leqslant C_{n}(1+|t|)$, it turns out to be coercive. Thus for any $n \geqslant 0$ we can find $u_{n} \in B_{n}$ solving the minimization problem

$$
J_{n}\left(u_{n}\right)=m_{n}=\inf _{u \in B_{n}} J_{n}(u) .
$$

Notice that by the choice of $\varepsilon_{n}$ and $\kappa_{n}$, we have

$$
J_{n}\left(u_{n}\right) \leqslant J_{n}\left(\tilde{u}_{n}\right) \leqslant J\left(\tilde{u}_{n}\right)+\varepsilon_{n}<J\left(u_{0}\right)=J_{n}\left(u_{0}\right) .
$$

We claim that there exists $\lambda_{n} \geqslant 0$ such that the following identity holds in $W^{-s, p^{\prime}}(\Omega)$ :

$$
(-\Delta)_{p}^{s} u_{n}+\lambda_{n}\left(u_{n}-u_{0}\right)^{p_{s}^{*}-1}=f_{n}\left(x, u_{n}\right)
$$

Indeed, recalling that $u_{n} \in B_{n}$, two cases may occur:

(a) If $\left\|u_{n}-u_{0}\right\|_{p_{s}^{*}}<\delta_{n}$, then by (3.2) and the continuous embedding $W_{0}^{s, p}(\Omega) \hookrightarrow L^{p_{s}^{*}}(\Omega)$, $u_{n}$ is a local minimizer of $J_{n}$ in $W_{0}^{s, p}(\Omega)$, hence $J_{n}^{\prime}\left(u_{n}\right)=0$. So, (3.4) holds with $\lambda_{n}=0$.

(b) If $\left\|u_{n}-u_{0}\right\|_{p_{s}^{*}}=\delta_{n}$, then we apply Lagrange's multipliers rule. Set for all $u \in W_{0}^{s, p}(\Omega)$

$$
I(u)=\frac{\left\|u-u_{0}\right\|_{p_{s}^{*}}^{p_{s}^{*}}}{p_{s}^{*}}, \quad \mathcal{M}_{n}=\left\{u \in W_{0}^{s, p}(\Omega): I(u)=\frac{\delta_{n}^{p_{s}^{*}}}{p_{s}^{*}}\right\},
$$

then $I \in C^{1}\left(W_{0}^{s, p}(\Omega)\right)$ and $\mathcal{M}_{n}$ is a $C^{1}$-manifold in $W_{0}^{s, p}(\Omega)$. By (3.2), $u_{n}$ is a global minimizer of $J_{n}$ on $\mathcal{M}_{n}$, so there exists $\lambda_{n} \in \mathbb{R}$ such that in $W^{-s, p^{\prime}}(\Omega)$

$$
J_{n}^{\prime}\left(u_{n}\right)+\lambda_{n} I^{\prime}\left(u_{n}\right)=0,
$$

which is equivalent to (3.4). Besides, by (3.2) again we have

$$
\lambda_{n}=-\frac{\left\langle J_{n}^{\prime}\left(u_{n}\right), u_{0}-u_{n}\right\rangle}{\left\langle I^{\prime}\left(u_{n}\right), u_{0}-u_{n}\right\rangle} \geqslant 0 .
$$

By construction we have that $u_{n} \rightarrow u_{0}$ in $L^{p_{s}^{*}}(\Omega)$, as $n \rightarrow \infty$. Moreover, by Lemma 2.1 and (3.4), we have $u_{n} \in L^{\infty}(\Omega)$. The next and most delicate step of the proof consists in proving that

$$
u_{n} \rightarrow u_{0} \quad \text { in } L^{\infty}(\Omega)
$$


Adding (1.3) and (3.4), for all $n \in \mathbb{N}$ we get in $W^{-s, p^{\prime}}(\Omega)$

$$
(-\Delta)_{p}^{s} u_{n}-(-\Delta)_{p}^{s} u_{0}+\lambda_{n}\left(u_{n}-u_{0}\right)^{p_{s}^{*}-1}=g_{n}\left(x, u_{n}-u_{0}\right),
$$

where for all $(x, t) \in \Omega \times \mathbb{R}$ we have set

$$
g_{n}(x, t):=f_{n}\left(x, t+u_{0}(x)\right)-f\left(x, u_{0}(x)\right) .
$$

By (1.4), we can find $C>0$ (independent of $n$ ) such that for all $n \in \mathbb{N}$

$$
\left|g_{n}(x, t)\right| \leqslant C\left(1+|t|^{p_{s}^{*}-1}\right) .
$$

We set $w_{n}=u_{n}-u_{0} \in W_{0}^{s, p}(\Omega) \cap L^{\infty}(\Omega)$ and test (3.6) with $w_{n}^{q} \in W_{0}^{s, p}(\Omega)$, for $q \geqslant 1$ :

$$
\begin{aligned}
& \left\langle(-\Delta)_{p}^{s} u_{n}-(-\Delta)_{p}^{s} u_{0}, w_{n}^{q}\right\rangle+\lambda_{n} \int_{\Omega}\left|w_{n}\right|^{p_{s}^{*}+q-1} d x=\int_{\Omega} g_{n}\left(x, w_{n}\right) w_{n}^{q} d x \\
& \leqslant C\left[\int_{\Omega}\left|w_{n}\right|^{q} d x+\int_{\Omega}\left|w_{n}\right|^{p_{s}^{*}+q-1} d x\right] .
\end{aligned}
$$

We now apply Lemma 2.3 and the continuous embedding $W_{0}^{s, p}(\Omega) \hookrightarrow L^{p_{s}^{*}}(\Omega)$ to get

$$
\begin{aligned}
{\left[\int_{\Omega}\left|w_{n}\right|^{\frac{p_{s}^{*}(p+q-1)}{p}} d x\right]^{\frac{p}{p_{s}^{*}}} } & \leqslant C\left\|w_{n}^{\frac{p+q-1}{p}}\right\|_{s, p}^{p} \\
& \leqslant C q^{p-1}\left\langle(-\Delta)_{p}^{s} u_{n}-(-\Delta)_{p}^{s} u_{0}, w_{n}^{q}\right\rangle,
\end{aligned}
$$

which, along with $\lambda_{n} \geqslant 0$ and (3.8), implies for all $n \in \mathbb{N}, q \geqslant 1$

$$
\left[\int_{\Omega}\left|w_{n}\right|^{\frac{p_{s}^{*}(p+q-1)}{p}} d x\right]^{\frac{p}{p_{s}^{*}}} \leqslant C q^{p-1}\left[\int_{\Omega}\left|w_{n}\right|^{q} d x+\int_{\Omega}\left|w_{n}\right|^{p_{s}^{*}+q-1} d x\right]
$$

with $C>0$ independent of $q$ and $n$. Next, we shall derive an iterative formula from (3.9). We define recursively an increasing sequence $\left(q_{j}\right)_{j}$ by setting

$$
q_{1}=1, \quad q_{j+1}=\frac{p_{s}^{*}\left(p+q_{j}-1\right)}{p},
$$

so $q_{j} \rightarrow \infty$ as $j \rightarrow \infty$. In particular, we can find $\bar{\jmath} \in \mathbb{N}$ such that

$$
\bar{q}=q_{\bar{\jmath}}>\frac{N\left(p_{s}^{*}-1\right)}{p s} .
$$

By (3.9) with $q=q_{j}$, we have for all $j, n \in \mathbb{N}$

$$
\left[\int_{\Omega}\left|w_{n}\right|^{q_{j+1}} d x\right]^{\frac{p}{p_{s}^{*}}} \leqslant C q_{j}^{p-1}\left[\int_{\Omega}\left|w_{n}\right|^{q_{j}} d x+\int_{\Omega}\left|w_{n}\right|^{p_{s}^{*}+q_{j}-1} d x\right] .
$$

We aim at absorbing the last integral into the left hand side. By Hölder's inequality we have

$$
\int_{\Omega}\left|w_{n}\right|^{p_{s}^{*}+q_{j}-1} d x \leqslant\left[\int_{\Omega}\left|w_{n}\right|^{p_{s}^{*}} d x\right]^{\frac{p_{s}^{*}-p}{p_{s}^{*}}}\left[\int_{\Omega}\left|w_{n}\right|^{\frac{p_{s}^{*}\left(p+q_{j}-1\right)}{p}} d x\right]^{\frac{p}{p_{s}^{*}}} .
$$

Since $w_{n} \rightarrow 0$ in $L^{p_{s}^{*}}(\Omega)$, for all $n \in \mathbb{N}$ big enough we may assume

$$
\left[\int_{\Omega}\left|w_{n}\right|^{p_{s}^{*}} d x\right]^{\frac{p_{s}^{*}-p}{p_{s}^{*}}} \leqslant \frac{1}{2 C \bar{q}^{p-1}} .
$$

So for all $j=1, \ldots \bar{\jmath}$ we have

which yields

$$
\left[\int_{\Omega}\left|w_{n}\right|^{q_{j+1}} d x\right]^{\frac{p}{p_{s}^{*}}} \leqslant C q_{j}^{p-1} \int_{\Omega}\left|w_{n}\right|^{q_{j}} d x+\frac{1}{2}\left[\int_{\Omega}\left|w_{n}\right|^{q_{j+1}} d x\right]^{\frac{p}{p_{s}^{*}}},
$$

$$
\left[\int_{\Omega}\left|w_{n}\right|^{q_{j+1}} d x\right]^{\frac{p}{p_{s}^{*}}} \leqslant 2 C q_{j}^{p-1} \int_{\Omega}\left|w_{n}\right|^{q_{j}} d x
$$


Iterating on (3.11) for $j=1, \ldots \bar{\jmath}$, we find $\bar{C}, \bar{\alpha}>0$ such that for all $n \in \mathbb{N}$ big enough

$$
\left\|w_{n}\right\|_{\bar{q}} \leqslant \bar{C}\left\|w_{n}\right\|_{1}^{\bar{\alpha}} \text {. }
$$

Since $\left(w_{n}\right)_{n}$ is bounded in $L^{1}(\Omega)$, it is so in $L^{\bar{q}}(\Omega)$ as well. Now recall (3.10) and set

$$
\bar{r}=\frac{\bar{q}}{p_{s}^{*}-1}>\frac{N}{p s}
$$

By (3.7) and Hölder's inequality we have

$$
\int_{\Omega}\left|g_{n}\left(x, w_{n}\right)\right|^{\bar{r}} d x \leqslant C \int_{\Omega}\left(1+\left|w_{n}\right|^{\bar{q}}\right) d x \leqslant C .
$$

Again we test (3.6) with $w_{n}^{q} \in W_{0}^{s, p}(\Omega) \cap L^{\infty}(\Omega), q \geqslant 1$. As above, by using Lemma 2.3 we get

$$
\begin{aligned}
{\left[\int_{\Omega}\left|w_{n}\right|^{\frac{p_{s}^{*}(p+q-1)}{p}} d x\right]^{\frac{p}{p_{s}^{*}}} } & \leqslant C q^{p-1} \int_{\Omega} g_{n}\left(x, w_{n}\right) w_{n}^{q} d x \\
& \leqslant C q^{p-1}\left[\int_{\Omega}\left|g_{n}\left(x, w_{n}\right)\right|^{\bar{r}} d x\right]^{\frac{1}{\bar{r}}}\left[\int_{\Omega}\left|w_{n}\right|^{q \bar{r}^{\prime}} d x\right]^{\frac{1}{\bar{r}^{\prime}}} \\
& \leqslant C q^{p-1}\left[\int_{\Omega}\left|w_{n}\right|^{\bar{r}^{\prime}} d x\right]^{\frac{1}{\bar{r}^{\prime}}},
\end{aligned}
$$

with $C>1$ independent of $n$ and $q$. By (3.10) we may set

$$
\gamma=\frac{p_{s}^{*}}{p \bar{r}^{\prime}}>1
$$

and define recursively two sequences $\left(p_{j}\right)_{j},\left(q_{j}\right)_{j}$ (different from the previous $\left.\left(q_{j}\right)_{j}\right)$ through

$$
p_{0}=p_{s}^{*}, \quad p_{j+1}=\gamma p_{j}+\frac{p_{s}^{*}(p-1)}{p}, \quad q_{j}=\frac{p_{j}}{\bar{r}^{\prime}} .
$$

So we have $p_{j}, q_{j} \rightarrow \infty$ as $j \rightarrow \infty$ and setting $q=q_{j}$ in (3.12), we have for all $n, j \in \mathbb{N}$

$$
\left[\int_{\Omega}\left|w_{n}\right|^{p_{j+1}} d x\right]^{\frac{p}{p_{s}^{*}}} \leqslant C q_{j}^{p}\left[\int_{\Omega}\left|w_{n}\right|^{p_{j}} d x\right]^{\frac{1}{\bar{r}^{\prime}}}
$$

(recall that $q_{j}>1$, hence $q_{j}^{p-1} \leqslant q_{j}^{p}$ ), which rephrases as the following recursive inequality:

$$
\int_{\Omega}\left|w_{n}\right|^{p_{j+1}} d x \leqslant\left(C q_{j}^{p}\right)^{\gamma \bar{r}^{\prime}}\left[\int_{\Omega}\left|w_{n}\right|^{p_{j}} d x\right]^{\gamma} .
$$

Iterating on (3.13) for $j \in \mathbb{N}$ and recalling that $q_{j} \sim \gamma^{j} / \bar{r}^{\prime}$ as $j \rightarrow \infty$, we have

$$
\begin{aligned}
\int_{\Omega}\left|w_{n}\right|^{p_{j}} d x & \leqslant \prod_{i=0}^{j-1}\left(C q_{i}^{p}\right)^{\gamma^{j-i} \bar{r}^{\prime}}\left[\int_{\Omega}\left|w_{n}\right|^{p_{0}} d x\right]^{\gamma^{j}} \\
& \leqslant C^{\gamma^{j}} \gamma^{p \bar{p}^{\prime} \sum_{i=0}^{j-1} i \gamma^{j-i}}\left[\int_{\Omega}\left|w_{n}\right|^{p_{s}^{*}} d x\right]^{\gamma^{j}},
\end{aligned}
$$

with an even bigger $C>1$ independent of $j, n$. Set

$$
S=\sum_{i=0}^{\infty} i \gamma^{-i}<\infty
$$

then we have for all $n, j \in \mathbb{N}$

$$
\int_{\Omega}\left|w_{n}\right|^{p_{j}} d x \leqslant C^{\gamma^{j}} \gamma^{\bar{r}^{\prime} S \gamma^{j}}\left[\int_{\Omega}\left|w_{n}\right|^{p_{s}^{*}} d x\right]^{\gamma^{j}}
$$


Note that, since $w_{n} \rightarrow 0$ in $L^{p_{s}^{*}}(\Omega)$, the integral on the right hand side is less than 1 for all $n \in \mathbb{N}$ big enough. Raising the last inequality to the power $1 / p_{j}$, we get

$$
\begin{aligned}
\left\|w_{n}\right\|_{p_{j}} & \leqslant C^{\frac{\gamma^{j}}{p_{j}}} \gamma^{p \bar{r}^{\prime} S \frac{\gamma^{j}}{p_{j}}}\left[\int_{\Omega}\left|w_{n}\right|^{p_{s}^{*}} d x\right]^{\frac{\gamma^{j}}{p_{j}}} \\
& \leqslant C^{\beta} \gamma^{p \bar{r}^{\prime} S \beta}\left[\int_{\Omega}\left|w_{n}\right|^{p_{s}^{*}} d x\right]^{\eta}
\end{aligned}
$$

where $\beta, \eta>0$ have been chosen such that for all $j \in \mathbb{N}$

$$
\eta<\frac{\gamma^{j}}{p_{j}}<\beta
$$

Summarizing, we find $C>1$ such that for all $n, j \in \mathbb{N}$ big enough

$$
\left\|w_{n}\right\|_{p_{j}} \leqslant C\left\|w_{n}\right\|_{p_{s}^{*}}^{\eta p_{s}^{*}}
$$

Letting $j \rightarrow \infty$ and recalling that $w_{n} \in L^{\infty}(\Omega)$, we have for all $n \in \mathbb{N}$ large enough

$$
\left\|w_{n}\right\|_{\infty} \leqslant C\left\|w_{n}\right\|_{p_{s}^{*}}^{\eta p_{s}^{*}}
$$

Finally, from $w_{n} \rightarrow 0$ in $L^{p_{s}^{*}}(\Omega)$ we infer $w_{n} \rightarrow 0$ in $L^{\infty}(\Omega)$ as well, thus proving (3.5).

We can now conclude the proof. For $n \in \mathbb{N}$ big enough, (3.4) rephrases as

$$
(-\Delta)_{p}^{s} u_{n}=f\left(x, u_{n}\right)-\lambda_{n}\left(u_{n}-u_{0}\right)^{p_{s}^{*}-1} \text { in } W^{-s, p^{\prime}}(\Omega)
$$

with $\lambda_{n} \geqslant 0$ (possibly $\lambda_{n} \rightarrow \infty$ ). As a consequence of (3.5), the sequence $\left(u_{n}\right)_{n}$ is bounded in $L^{\infty}(\Omega)$, so by (1.4) we see that $\left(f\left(\cdot, u_{n}\right)\right)_{n}$ is uniformly bounded as well. To go further we need a uniform bound on $\left(\lambda_{n}\left(u_{n}-u_{0}\right)^{p_{s}^{*}-1}\right)_{n}$. Testing again (3.6) with $w_{n}^{q}$ (with $w_{n}=u_{n}-u_{0}$, $q \geqslant 1$ ) and applying Lemma 2.3, we get for all $n \in \mathbb{N}$ big enough

$$
\begin{aligned}
\lambda_{n} \int_{\Omega}\left|w_{n}\right|^{p_{s}^{*}+q-1} d x & \leqslant \int_{\Omega} g\left(x, u_{n}\right) w_{n}^{q} d x \\
& \leqslant C \int_{\Omega}\left|w_{n}\right|^{q} d x \\
& \leqslant C\left[\int_{\Omega}\left|w_{n}\right|^{p_{s}^{*}+q-1} d x\right]^{\frac{q}{p_{s}^{*}+q-1}}|\Omega|^{\frac{p_{s}^{*}-1}{p_{s}^{*}+q-1}}
\end{aligned}
$$

(with $C>0$ independent of $n, q$ ), which implies

$$
\lambda_{n}\left\|w_{n}\right\|_{p_{s}^{*}+q-1}^{p_{s}^{*}-1} \leqslant C|\Omega|^{\frac{p_{s}^{*}-1}{p_{s}^{*}+q-1}} .
$$

Letting $q \rightarrow \infty$, we have

$$
\lambda_{n}\left\|w_{n}\right\|_{\infty}^{p_{s}^{*}-1} \leqslant C
$$

i.e., $\left(\lambda_{n}\left(u_{n}-u_{0}\right)^{p_{s}^{*}-1}\right)_{n}$ is a bounded sequence in $L^{\infty}(\Omega)$. Then, (3.14) and Lemma 2.2 imply that $\left(u_{n}\right)_{n}$ is bounded in $C_{s}^{\alpha}(\bar{\Omega})$. By the compact embedding $C_{s}^{\alpha}(\bar{\Omega}) \hookrightarrow C_{s}^{0}(\bar{\Omega})$, passing to a subsequence we have $u_{n} \rightarrow u_{0}$ in $C_{s}^{0}(\bar{\Omega})$. So, for all $n \in \mathbb{N}$ big enough we have $\left\|u_{n}-u_{0}\right\|_{C_{s}^{0}} \leqslant \sigma$. On the other hand, being $\left(u_{n}\right)_{n}$ bounded in $L^{\infty}(\Omega)$, for $n$ large enough we have $J_{n}\left(u_{n}\right)=J\left(u_{n}\right)$, so that by (3.3) we have $J\left(u_{n}\right)<J\left(u_{0}\right)$. Thus we reached a contradiction to $(i i)$, and $(i)$ is proved. 


\section{An APPLiCAtion}

To conclude we just want to give an example of how our result works, presenting a nonlinear extension of [8, Theorem 3.3]. We make on the reaction $f$ in problem (1.3) the following assumptions:

$\mathbf{H} f: \Omega \times \mathbb{R} \rightarrow \mathbb{R}$ is a Carathéodory map such that

(i) $|f(x, t)| \leqslant C_{0}\left(1+|t|^{q-1}\right)$ for a.e. $x \in \Omega$, all $t \in \mathbb{R}\left(C_{0}>0, q \in\left(p, p_{s}^{*}\right)\right)$;

(ii) $f(x, t) t \geqslant 0$ for a.e. $x \in \Omega$, all $t \in \mathbb{R}$;

(iii) $\limsup _{|t| \rightarrow \infty} \frac{F(x, t)}{|t|^{p}} \leqslant 0$ uniformly for a.e. $x \in \Omega$;

(iv) $\liminf _{t \rightarrow 0} \frac{F(x, t)}{|t|^{p}}>\frac{\lambda_{2}}{p}$ uniformly for a.e. $x \in \Omega$.

Here $\lambda_{2}>0$ denotes the second (variational) eigenvalue of $(-\Delta)_{p}^{s}$ in $W_{0}^{s, p}(\Omega)$ (see [3] for details). Under these assumptions, we prove the following multiplicity result for problem (1.3):

Theorem 4.1. Let $\mathbf{H}$ be satisfied. Then, problem (1.3) admits at least three nontrivial solutions.

Proof. We just sketch the proof, referring to [8] for details. First we introduce two truncated reactions and their primitives, defined for all $(x, t) \in \Omega \times \mathbb{R}$ by

$$
f_{ \pm}(x, t)=f\left(x, \pm t^{ \pm}\right), \quad F_{ \pm}(x, t)=\int_{0}^{t} f_{ \pm}(x, \tau) d \tau
$$

(here $t^{ \pm}=\max \{ \pm t, 0\}$ ), and note that $f_{ \pm}$are Carathéodory with subcritical growth due to $\mathbf{H}(i)$ (ii). We introduce the corresponding truncated energy functionals $J_{ \pm} \in C^{1}\left(W_{0}^{s, p}(\Omega)\right)$ defined by

$$
J_{ \pm}(u)=\frac{\|u\|_{s, p}^{p}}{p}-\int_{\Omega} F_{ \pm}(x, u) d x
$$

By $\mathbf{H}(i i i), J_{+}$is coercive and thus it admits a global minimizer $u_{+} \in W_{0}^{s, p}(\Omega)$. Using $\mathbf{H}(i v)$, we easily see that $u_{+} \neq 0$. Clearly, $u_{+}$is a weak solution of the auxiliary problem

$$
\begin{cases}(-\Delta)_{p}^{s} u=f_{+}(x, u) & \text { in } \Omega \\ u=0 & \text { in } \mathbb{R}^{N} \backslash \Omega,\end{cases}
$$

hence by Lemmas 2.1, 2.2 we have $u_{+} \in C_{s}^{0}(\bar{\Omega})$. Besides, by the fractional $p$-Laplacian Hopf's lemma (see [7, Theorem 1.5]), we have uniformly for all $x \in \partial \Omega$

$$
\lim _{\Omega \ni y \rightarrow x} \frac{u_{+}(y)}{\mathrm{d}_{\Omega}^{s}(y)}>0 .
$$

Thus, $u_{+}$lies in the interior of the positive order cone $C_{s}^{0}(\bar{\Omega})_{+}$of $C_{s}^{0}(\bar{\Omega})$ (see [15, Lemma 5.1]). Since $J_{+}=J$ on $C_{s}^{0}(\bar{\Omega})_{+}$, we see that $u_{+}$is a local minimizer of $J$ in the $C_{s}^{0}(\bar{\Omega})$-topology. By Theorem 1.1, $u_{+}$is as well a local minimizer of $J$ in the $W_{0}^{s, p}(\Omega)$-topology.

Arguing similarly on $J_{-}$, we detect a local minimizer $u_{-} \in-\operatorname{int}\left(C_{s}^{0}(\bar{\Omega})_{+}\right)$of $J$. Plus, $J$ satisfies the Palais-Smale condition, hence by the mountain pass theorem it admits one more critical point $\tilde{u} \in W_{0}^{s, p}(\Omega)$. Exploiting condition $\mathbf{H}(i v)$ and the variational characterization of the second eigenvalue $\lambda_{2}$ (see [3, Theorem 5.3]), we see that $\tilde{u} \neq 0$. Finally, we use Lemmas 2.1, 2.2 to deduce that $\tilde{u} \in C_{s}^{0}(\bar{\Omega})$.

All in all, $u_{+}, u_{-}, \tilde{u} \in W_{0}^{s, p}(\Omega) \cap C_{s}^{0}(\bar{\Omega}) \backslash\{0\}$ are three nontrivial solutions of (1.3). 
Acknowledgement. All authors are members of GNAMPA (Gruppo Nazionale per l'Analisi Matematica, la Probabilità e le loro Applicazioni) of INdAM (Istituto Nazionale di Alta Matematica 'Francesco Severi'). A. Iannizzotto and S. Mosconi are supported by the grant PRIN n. 2017AYM8XW: Nonlinear Differential Problems via Variational, Topological and Set-valued Methods. A. Iannizzotto is also supported by the research project Integro-differential Equations and nonlocal Problems, funded by Fondazione di Sardegna (2017), S. Mosconi by the grant PdR 2018-2020 - linea di intervento 2: Metodi Variazionali ed Equazioni Differenziali of the University of Catania.

\section{REFERENCES}

[1] A. Ambrosetti, H. Brézis, G. Cerami, Combined effects of concave-convex nonlinearities in some elliptic problems, J. Funct. Anal. 122 (1994) 519-543.

[2] B. Barrios, E. Colorado, R. Servadei, F. Soria, A critical fractional equation with concave-convex power nonlinearities, Ann. Inst. H. Poincaré Anal. Non Linéaire 32 (2015) 875-900.

[3] L. Brasco, E. Parini, The second eigenvalue of the fractional p-Laplacian, Adv. Calc. Var. 9 (2016) 323-355.

[4] H. Brezis, L. Nirenberg, $H^{1}$ versus $C^{1}$ local minimizers, C. R. Acad. Sci. Paris Sér. I 317 (1993) 465-472.

[5] F. Brock, L. Iturriaga, P. Ubilla, A multiplicity result for the $p$-Laplacian involving a parameter, Ann. Henri Poincaré 9 (2008) 1371-1386.

[6] W. Chen, S. Mosconi, M. Squassina, Nonlocal problems with critical Hardy nonlinearity, J. Funct. Anal. 275 (2018) 3065-3114.

[7] L.M. Del Pezzo, A. QuaAs, A Hopf's lemma and a strong minimum principle for the fractional $p$ Laplacian, J. Differential Equations 263 (2017) 765-778.

[8] F.G. Düzgün, A. IAnnizzotto, Three nontrivial solutions for nonlinear fractional Laplacian equations, Adv. Nonlinear Analysis 7 (2018) 211-226.

[9] X. FAn, On the sub-supersolution method for $p(x)$-Laplacian equations, J. Math. Anal. Appl. 330 (2007) 665-682.

[10] D.G. de Figueiredo, J.P. Gossez, P. Ubilla, Local 'superlinearity' and 'sublinearity' for the $p$-Laplacian, J. Funct. Anal. 257 (2009) 721-752.

[11] S. Frassu, Nonlinear Dirichlet problem for the nonlocal anisotropic operator $L_{K}$, Comm. Pure Appl. Anal. 18 (2019) 1847-1867.

[12] Y. Fu, P. PuCCI, Multiplicity existence for sublinear fractional Laplacian problems, Appl. Anal. 96 (2017) 1497-1508.

[13] J.P. Garcìa Azorero, I. Peral Alonso, J.J. Manfredi, Sobolev versus Hölder local minimizers and global multiplicity for some quasilinear elliptic equations, Commun. Contemp. Math. 2 (2000) 385-404.

[14] L. Gasiński, N.S. Papageorgiou, Multiple solutions for nonlinear coercive problems with a nonhomogeneous differential operator and a nonsmooth potential, Set-Valued Var. Anal. 20 (2012) 417-443.

[15] A. Iannizzotto, S. Liu, K. Perera, M. Squassina, Existence results for fractional $p$-Laplacian problems via Morse theory, Adv. Calc. Var. 9 (2016) 101-125.

[16] A. Iannizzotto, S. Mosconi, M. Squassina, $H^{s}$ versus $C^{0}$-weighted minimizers, Nonlinear Differ. Equ. Appl. 22 (2015) 477-497.

[17] A. Iannizzotto, S. Mosconi, M. Squassina, Global Hölder regularity for the fractional $p$-Laplacian, Rev. Mat. Iberoam. 32 (2016) 1353-1392.

[18] A. Iannizzotto, S. Mosconi, M. Squassina, A note on global regularity for the weak solutions of fractional p-Laplacian equations, Rend. Lincei Mat. Appl. 27 (2016) 15-24.

[19] A. Innnizzotto, S. Mosconi, M. Squassina, Fine boundary regularity for the fractional p-Laplacian, (preprint).

[20] A. Innnizzotto, N.S. Papageorgiou, Existence of three nontrivial solutions for nonlinear Neumann hemivariational inequalities, Nonlinear Anal. 70 (2009) 3285-3297.

[21] S. JAROHS, Strong comparison principle for the fractional $p$-Laplacian and applications to starshaped rings, Adv. Nonlinear Studies 18 (2018) 691-704.

[22] G. M. Lieberman, Boundary regularity for solutions of degenerate elliptic equations, Nonlinear Anal. 12 (1988) 1203-1219.

[23] J. LiU, S. LiU, The existence of multiple solutions to quasilinear elliptic equations, Bull. London Math. Soc. 37 (2005) 592-600. 
[24] S. Mosconi, M. Squassina, Recent progresses in the theory of nonlinear nonlocal problems, Bruno Pini Mathematical Analysis Sem. 7 (2016) 147-164.

[25] X. Ros-Oton, J. Serra, The Dirichlet problem for the fractional Laplacian: regularity up to the boundary, J. Math. Pures Appl. 101 (2014) 275-302.

[26] N. S. Ustinov, Multiplicity of positive solutions to the boundary-value problems for fractional Laplacians, J. Math. Sci. 236 (2019), 236: 446.

[27] Z.Q. Wang, On a superlinear elliptic equation, Ann. Inst. H. Poincaré Anal. Non Linéaire 8 (1991) 43-57.

(A. Iannizzotto) Department of Mathematics and Computer Science UNIVERSITÀ DEGLI STUdI DI CAGLIARI

Viale L. Merello 92, 09123 Cagliari, Italy

E-mail address: antonio.iannizzotto@unica.it

(S. Mosconi) Dipartimento di Matematica e Informatica Università degli Studi di Catania

Viale A. Doria 6, 95125 Catania, Italy

E-mail address: mosconi@dmi.unict.it

(M. Squassina) Dipartimento di Matematica e Fisica

Università Cattolica del SaCro Cuore

Via Dei Musei 41, 25121 Brescia, ItAly

E-mail address: marco.squassina@unicatt.it 